\title{
Automatic estimation of the aortic lumen geometry by ellipse tracking
}

\author{
Pablo G. Tahoces ${ }^{1}$ (1) $\cdot$ Luis Alvarez $^{2}$ (D) Esther González ${ }^{2} \cdot$ Carmelo Cuenca $^{2} \cdot$ Agustín Trujillo $^{2}$. \\ Daniel Santana-Cedrés ${ }^{2}$ - Julio Esclarín ${ }^{2} \cdot$ Luis Gomez $^{2} \cdot$ Luis Mazorra $^{2} \cdot$ Miguel Alemán-Flores $^{2} \cdot$ José M. Carreira $^{3}$
}

Received: 30 January 2018 / Accepted: 12 September 2018 / Published online: 22 September 2018

(c) CARS 2018

\begin{abstract}
Purpose The shape and size of the aortic lumen can be associated with several aortic diseases. Automated computer segmentation can provide a mechanism for extracting the main features of the aorta that may be used as a diagnostic aid for physicians. This article presents a new fully automated algorithm to extract the aorta geometry for either normal (with and without contrast) or abnormal computed tomography (CT) cases.

Methods The algorithm we propose is a fast incremental technique that computes the 3D geometry of the aortic lumen from an initial contour located inside it. Our approach is based on the optimization of the 3D orientation of the cross sections of the aorta. The method uses a robust ellipse estimation algorithm and an energy-based optimization technique to automatically track the centerline and the cross sections. The optimization involves the size and eccentricity of the ellipse which best fits the aorta contour on each cross-sectional plane. The method works directly on the original CT and does not require a prior segmentation of the aortic lumen. We present experimental results to show the accuracy of the method and its ability to cope with challenging CT cases where the aortic lumen may have low contrast, different kinds of pathologies, artifacts, and even significant angulations due to severe elongations.

Results The algorithm correctly tracked the aorta geometry in 380 of $385 \mathrm{CT}$ cases. The mean of the dice similarity coefficient was 0.951 for aorta cross sections that were randomly selected from the whole database. The mean distance to a manually delineated segmentation of the aortic lumen was $0.9 \mathrm{~mm}$ for sixteen selected cases.

Conclusions The results achieved after the evaluation demonstrate that the proposed algorithm is robust and accurate for the automatic extraction of the aorta geometry for both normal (with and without contrast) and abnormal CT volumes.
\end{abstract}

Keywords Aorta $\cdot$ Ellipse tracking $\cdot$ Centerline $\cdot$ Cross section $\cdot$ CT images

\section{Introduction}

The practice of evidence-based medicine [1] has become a reality, with the publication of guidelines for the diagnosis and management of patients of different diseases from the results achieved in several meta-studies. In many cases, these guidelines employ quantitative imaging biomarkers of great usefulness for providing evidence-based decisions.

Pablo G. Tahoces

pablo.tahoces@usc.es

1 Department of Electronics and Computer Science, Universidad de Santiago de Compostela, Santiago de Compostela, Spain

2 CTIM, Universidad de Las Palmas de Gran Canaria, Las Palmas de Gran Canaria, Spain

3 Complejo Hospitalario Universitario de Santiago (CHUS), Santiago de Compostela, Spain
Thereby, the introduction of new computer tools that can provide robust, reliable, accurate and reproducible values of such biomarkers has become a fruitful area of development.

In particular, the presence of aneurysms, dissections, mural thrombi, or elongations are currently considered the main anomalies of the thoracic aorta [2], and the use of images from CT scanners has been extensively employed to study these. Moreover, the correct measurement of distances, the presence of different textures, or the characterization of shapes are some of the main tasks to be addressed. The development of fully automated tools that can be applied to a broad variety of volumetric chest $\mathrm{CT}$ scans is currently a great challenge to be faced.

The first step in the development of such automated tools is the correct segmentation of the aorta from the whole CT volume [3]. Even though this task can be appeared to be relatively simple for contrast $\mathrm{CT}$ scans of normal cases, the 
presence of artifacts and abnormalities, or the use of noncontrast CT scans make the standard 3D segmentation, based on intensity or even edge information, fail due to the presence of neighboring pixels with similar intensity, which leads to leakage as the algorithm evolves.

Several algorithms for automated aorta segmentation from $\mathrm{CT}$ volume data have been previously developed. In general, the Hough Transform has been employed to detect a first slice, from which either a seed point, or an initial contour, are taken. From there, several approaches have been proposed. Wang et al. proposed an iterative method based on building a 2-D region for segmenting the ascending aorta [4]. Martinez et al. [5] employed active contours to delineate the aorta contour on each slice. However, such approaches were developed for contrast-enhanced CT scans, where there is a significant difference between the aorta and the surrounding tissues.

If no contrast is injected, the evolution of the algorithms is more challenging because the vessels are not enhanced from the background. To address this drawback, and to avoid the leakages, some approaches that rely on a priori shaped models are employed. However, the development of a shaped model for the aorta requires a large dataset of manually labeled training examples because the aorta can adopt multiple shapes, mainly from abnormal cases. This is the reason why most of the previous developments based on this idea use regular shapes such as the circle to model the aorta and perform the tracking by adapting the model to the orientation of its orthogonal cross section. This is the case for Kurugol et al. [6], where ascending and descending aorta were modeled as tubular shapes and the arch section as a torus, but in any case, preserving the circular shape for cross sections. Dasgupta et al. [7] proposed the use of morphological geodesic active contours to segment the aorta. The aorta boundary for both ascending and descending aorta was located by using a circular Hough Transform. Xie et al. [8] used a cylinder-tracking algorithm that follows the shape of the aorta by adapting the length of such cylinder to the aortic diameter and curvature. As in the two previous works, the first aorta boundary was automatically extracted in the slice where the carina is located. Furthermore, Krissian et al. [9] and Frangi et al. [10] used diffusion filters to enhance the contrast of vessels and improved the performance of vessel segmentation.

In this paper, we introduce an extension of the method described by Alvarez et al. [11], presented at the MICCAI Conference in 2017, to address the problem of estimating the aortic lumen in CT volumes. The main contribution consists in the development of a fully automated method to estimate the aortic lumen geometry, which relies on a tracking procedure of the aorta cross sections. Such cross sections are modeled by using ellipses oriented in the 3D space according to planes transversal to the aorta. To perform this task, the estimation of the aortic lumen geometry is formulated as an energy minimization problem, where we optimize the orientation in the space of the cross-sectional planes, as well as the ellipse parameters in these planes. To compute the ellipses, we have followed the method proposed in [12]. However, because we also have to deal with the optimization of the orientation of the cross-sectional planes, a more complex energy minimization formulation was required.

Potential applications of these methods are the extraction of morphology characteristics for detection of diseases [13], patient follow-up, surgery planning [14], or even as biomarkers in order to understand cardiovascular diseases [15]. Therefore, the method has to be accurate (the shape of the lumen has to be quite similar to the cross section obtained), and robust (the whole volume of the aorta has to be tracked without leakages, even in the presence of artifacts or diseases). In this way, an extensive experimental study that demonstrates the robustness and accuracy of this new technique is also presented in this paper, including the software and the dataset used as experimental setup on the Web site http://www.ctim.es/demo110/.

\section{Automatic tracking of the aortic lumen geom- etry}

The main goal of this paper is to design a new method that, by taking a 3D thoracic CT volume as input, allows extracting the 3D tubular structure of the aortic surface automatically, even in cases where the morphology of that structure has a certain degree of tortuosity. This occurs for the aortic arch, or in cases where the presence of severe elongations or mural thrombi transforms the cylindrical shape of the aorta into a more complex morphology.

The method can be described as a three-step process: First, the procedure starts from an initial circle that is automatically estimated in one of the slices of the CT volume. This can be located in either the ascending or descending aorta. The image intensity value of the $3 \mathrm{D}$ volume is then truncated using two threshold values to focus the analysis on the aorta lumen and the surrounding areas. Finally, the aorta centerline, the cross sections, and the ellipses fitting the aorta lumen are tracked in both upward and downward directions from the initial contour. The main tool used is an ellipse tracking algorithm, introduced in [12], and based on the minimization of the following energy:

$E_{1}(C, \mathcal{I}, \sigma)=\frac{1}{|C|} \oint_{C} \nabla G_{\sigma} * \mathcal{I}(C(s)) \cdot \bar{n}(s) \mathrm{d} s$,

where $\mathcal{I}$ is a $2 D$ image (the aorta cross section in our case), $C$ represents the ellipse, $G_{\sigma}$ is a Gaussian convolution kernel with standard deviation $\sigma$, and $\bar{n}$ represents the normal to the ellipse contour. In this way, the above line integral measures 
the contrast of the convolved image along the ellipse and the local minimum of such energy is attained for ellipses fitting high-contrast contours.

\section{Location of the first contour}

It is well known that, due to its tubular shape, when the orientation of the aorta is orthogonal to a CT slice, it can be approximated by a circle. For this reason, a circle Hough Transform is employed as a first step for the detection of an initial contour for our algorithm. However, several other structures, such as the trachea or the backbone also appear as circular shapes in those CT slices. To avoid the selection of circles that are present in the slices but do not correspond to vascular structures, a more complex procedure is required. To address this problem, we use the algorithm described in [16]. Briefly, for each slice $I^{z}$ of the 3D volume, a circle Hough Transform is computed. The most voted circle $C^{z}$, with center $\left(c_{x}^{z}, c_{y}^{z}\right)^{T}$ and radius $R^{z}$, is then collected for each slice. Next, an optimal circle, $C^{z \text { opt }}$, is selected from $\left\{C^{z}\right\}$, based on a $Q^{z}$ quality value that is defined for each of the $C^{z}$ circles as follows:

$$
Q^{z}=\frac{V^{z}}{\operatorname{Md}\left\{V^{z}\right\}}-\frac{E^{z}}{\operatorname{Md}\left\{E^{z}\right\}}-\frac{\sigma^{z}}{\operatorname{Md}\left\{\sigma^{z}\right\}}-\frac{D^{z}}{\operatorname{Md}\left\{D^{z}\right\}},
$$

where $\operatorname{Md}\{$.$\} represents the median estimator, V^{z}$ is the voting score provided by the Hough Transform, $\sigma^{z}$ is the standard deviation of the image intensity values inside the circle, $D^{z}$ is the distance between circle centers for consecutive slices $\left(c_{x}^{z}, c_{y}^{z}\right)^{T}$ and $\left(c_{x}^{z+1}, c_{y}^{z+1}\right)^{T}$, and $E^{z}$ is the following circle energy introduced in [17]:

$$
\begin{aligned}
E(R, \bar{c})= & \frac{1}{2 \pi} \int_{0}^{2 \pi} \nabla I_{\sigma}(C(\theta)) \cdot \bar{n}(\theta) \mathrm{d} \theta \\
& +\alpha_{-}\left(\frac{\iint_{A_{-}}\left(I_{\sigma}(C(\theta))-I_{-}\right)^{2} r \mathrm{~d} r \mathrm{~d} \theta}{\left|A_{-}\right|}\right)^{\frac{1}{2}} \\
& +\alpha_{+}\left(\frac{\iint_{A_{+}}\left(I_{\sigma}(C(\theta))-I_{+}\right)^{2} r \mathrm{~d} r \mathrm{~d} \theta}{\left|A_{+}\right|}\right)^{\frac{1}{2}},
\end{aligned}
$$
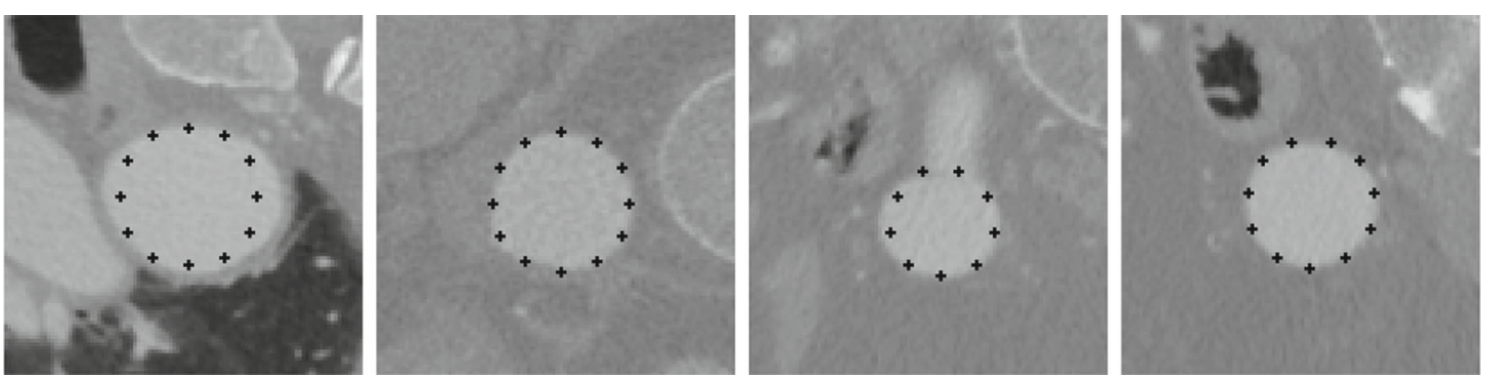

where $C(\theta)=\left(c_{x}+R \cdot \cos (\theta), c_{y}+R \cdot \sin (\theta)\right)^{T}, I_{\sigma}$ is the original image convolved with a Gaussian kernel, $\alpha_{-}, \alpha_{+} \geq$ $0, A_{-}, A_{+}$are annuli on both sides of the circle contour, and $I_{-}, I_{+}$are the average of $I_{\sigma}$ in $A_{-}$and $A_{+}$, respectively.

Once a reference circle $C^{z_{\text {opt }}}$ has been estimated by maximizing the quality criterion (2), we obtain, by similarity with this one, a collection of circles that could be used as well as initial contours. Figure 1 shows some of such circles for the same CT scan obtained in different slices. If the tracking of the aorta is stopped before the heart is reached, the method automatically detects this problem and starts from a new initial contour. This usually happens when the aortic arch is being tracked. If the algorithm is not able to fully track the aortic arch, we recomputed the aorta centerline by using another circle of that collection $\left\{C^{z_{i}}\right\}$ as initial contour. This is automatically performed by checking the distance between the position of the last centerline point obtained and the centerline point where the $z$ coordinate attains its maximum. If this distance is too short, a new initial contour from $\left\{C^{z_{i}}\right\}$ is taken.

\section{Estimation of the thresholds}

Following [16], from the collection of circles obtained above $\left\{C^{z_{i}}\right\}$, a kernel density estimation (KDE) of the distributions of the intensities inside and outside the aorta is computed (Fig. 2). Percentiles of the obtained distributions are used for estimating two thresholds $P_{\text {out }}$ and $P_{\text {in }}$ that are employed to perform a basic image truncation. That is, we update the CT volume intensity $I(x, y, z)$ in the following way:

$$
I(x, y, z)=\left\{\begin{array}{ll}
I(x, y, z) & \text { if } I(x, y, z) \in\left[P_{\text {out }}, P_{\text {in }}\right] \\
P_{\text {out }} & \text { if } I(x, y, z)<P_{\text {out }} \\
P_{\text {in }} & \text { if } I(x, y, z)>P_{\text {in }}
\end{array} .\right.
$$

The main goal of this truncation procedure is to exclude dark areas (due to the presence of air) or bright spots (due to the presence of calcifications, metallic structures, or even accumulations of physiological contrast) from the vicinity of the aorta (Fig. 3). These kinds of artifacts could affect the performance of the tracking algorithm that is described below.

Fig. 1 Initial contours obtained automatically using the method proposed in [16] 


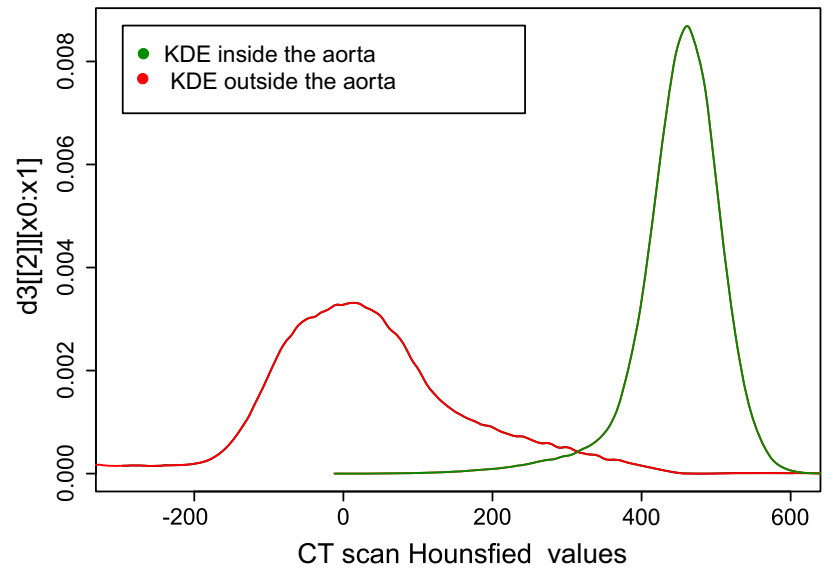

Fig. $2 \mathrm{KDE}$ of the density probability distributions inside and outside the aorta vessel obtained using the method proposed in [16]

\section{Tracking of the aorta}

As pointed out above, the aortic contour usually approaches a circular shape. However, on certain occasions this circular shape degenerates and it is more suitable to model these contour as an ellipse. Thus, for instance, when the tortuosity of the aorta is high and there are large angulations, the aorta cross section can be far away from the circular shape (Fig. 4). To deal with these kinds of drawbacks, we propose the following algorithm, based on ellipse tracking [11].

Let $I: \Omega \subset R^{3} \rightarrow R$ be a 3D image, $\mathbf{c}=\left(c_{x}, c_{y}, c_{z}\right)^{T} \in$ $R^{3}$, and $\alpha, \beta \in R$. We define the $2 D$ image $I_{\mathbf{c}}^{\alpha, \beta}(x, y)$ as

$$
\begin{aligned}
& I_{\mathbf{c}}^{\alpha, \beta}(x, y) \\
& =I(\left(\begin{array}{l}
c_{x} \\
c_{y} \\
c_{z}
\end{array}\right)+\underbrace{\left(\begin{array}{ccc}
\cos \alpha & 0 & \sin \alpha \\
-\sin \alpha \sin \beta & \cos \beta & \cos \alpha \sin \beta \\
-\cos \beta \sin \alpha-\sin \beta & \cos \alpha \cos \beta
\end{array}\right)}_{\mathbf{R}}\left(\begin{array}{l}
x \\
y \\
0
\end{array}\right))
\end{aligned}
$$

$I_{\mathbf{c}}^{\alpha, \beta}(x, y)$ represents the intersection of the $3 D \mathrm{CT}$ volume with the plane that contains the point $\mathbf{c}$ and is orthogonal to $\mathbf{n}=(\sin \alpha, \cos \alpha \sin \beta, \cos \alpha \cos \beta)^{T}$. In Fig. 5, we illustrate the shape of a cross-sectional plane. We denote by $C_{\mathbf{c}, \sigma}^{\alpha, \beta}$ the ellipse which optimizes the energy (1) for such an image.

To estimate the best orientation of the cross-sectional plane, a new energy is defined:

$$
\begin{aligned}
E(\alpha, \beta, \mathbf{c})= & w_{1} E_{1}\left(C_{\mathbf{c}, \sigma}^{\alpha, \beta}\right)+w_{2} \underbrace{\sqrt{\operatorname{Area}\left(C_{\mathbf{c}, \sigma}^{\alpha, \beta}\right)}}_{E_{2}\left(C_{\mathbf{c}, \sigma}^{\alpha, \beta}\right)} \\
& +w_{3} \underbrace{\operatorname{Eccentricity}\left(C_{\mathbf{c}, \sigma}^{\alpha, \beta}\right)}_{E_{3}\left(C_{\mathbf{c}, \sigma}^{\alpha, \beta}\right)},
\end{aligned}
$$

where $w_{1}, w_{2}, w_{3} \geq 0$. The above energy $E(\alpha, \beta, \mathbf{c})$ is the balance of three terms: $E_{1}\left(C_{\mathbf{c}, \sigma}^{\alpha, \beta}\right)$, given by (1), a quality factor related with the existence of high-contrast values between the aorta lumen and the surrounding region. $E_{2}\left(C_{\mathbf{c}, \sigma}^{\alpha, \beta}\right)$, that penalizes ellipses with a large area, and $E_{3}\left(C_{\mathbf{c}, \sigma}^{\alpha, \beta}\right)$, which in turns, penalizes a large value of the ellipse eccentricity. In order to set proper values for the weight parameters $w_{i}$, we seek to balance the three terms of the energy. It must be noted that, in the CT images, $E_{1}$ is proportional to the intensity values of the image, close to several hundreds. $E_{2}$ (square root of the area of the ellipse which fits the aorta cross section) is approximately a few tens of units, and $E_{3}$ (eccentricity) is in the range $[0,1)$. In all the experiments performed, the following values have been used: $w_{1}=0.1, w_{2}=1$ and $w_{3}=10$.

To track the centerline point $\mathbf{c}_{k}$, the orientation of the cross-sectional plane $\left(\alpha_{k}, \beta_{k}\right)$ and the ellipse in each cross section $C_{\mathbf{c}_{k}, \sigma}^{\alpha_{k}, \beta_{k}}$, the following iterative procedure is performed:

1. An initial estimation of $\mathbf{c}_{k}$ is computed as

$\mathbf{c}_{k}=\mathbf{c}_{k-1}+h \cdot \mathbf{n}_{k-1}$,

where $h$ is a discretization step (in the experiments we use $h$ as the minimum of the image voxel edge lengths ).

2. $\left(\alpha_{k}, \beta_{k}\right)$ and $C_{\mathbf{c}_{k}, \sigma}^{\alpha_{k}, \beta_{k}}$ are computed by minimizing energy $E\left(\alpha, \beta, \mathbf{c}_{k}\right)$ with respect to $\alpha, \beta$, that is

$$
\left(\alpha_{k}, \beta_{k}\right)=\underbrace{\arg \min }_{\alpha, \beta} E\left(\alpha, \beta, \mathbf{c}_{k}\right)
$$

The minimization is performed by a Newton-Raphsontype algorithm using the previous values $\left(\alpha_{k-1}, \beta_{k-1}\right)$ as initial guess for $\left(\alpha_{k}, \beta_{k}\right)$.

3. $\mathbf{c}_{k}$ is updated by applying the isometry defined in (7) to the center of the ellipse $C_{\mathbf{c}_{k}, \sigma}^{\alpha_{k}, \beta_{k}}$ in the following way:

$\mathbf{c}_{k}=\mathbf{c}_{k}+\mathbf{R} \cdot\left(x_{c}, y_{c}, 0\right)^{T}$,

where $\left(x_{c}, y_{c}\right)^{T}$ is the center of the ellipse $C_{\mathbf{c}_{k}, \sigma}^{\alpha_{k}, \beta_{k}}$.

4. The orthogonal direction $\mathbf{n}_{k}$ to the cross-sectional plane is computed as

$\mathbf{n}_{k}= \pm\left(\sin \alpha_{k}, \cos \alpha_{k} \sin \beta_{k}, \cos \alpha_{k} \cos \beta_{k}\right)^{T}$,

where the sign of $\mathbf{n}_{k}$ is fixed in such a way that $\mathbf{n}_{k} \cdot \mathbf{n}_{k-1}>$ 0 .

The same procedure is used to track the aorta upward or downward from the initial contour. This is accomplished by changing $h$ by $-h$ in the step 1 . 

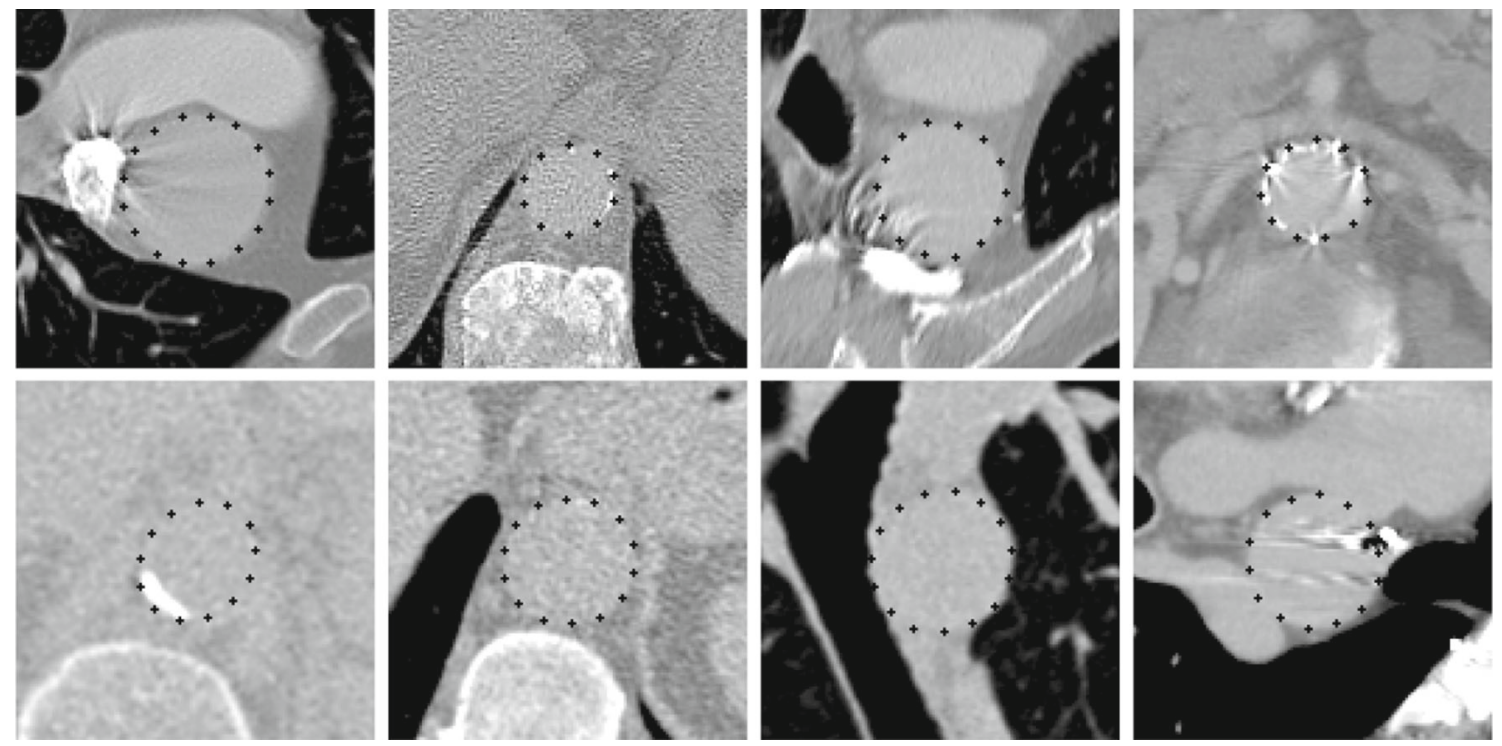

Fig. 3 Challenging cases from the LIDC database. In spite of the poor contrast of the images and the presence of artifacts, the proposed method is able to compute cross sections and ellipses properly
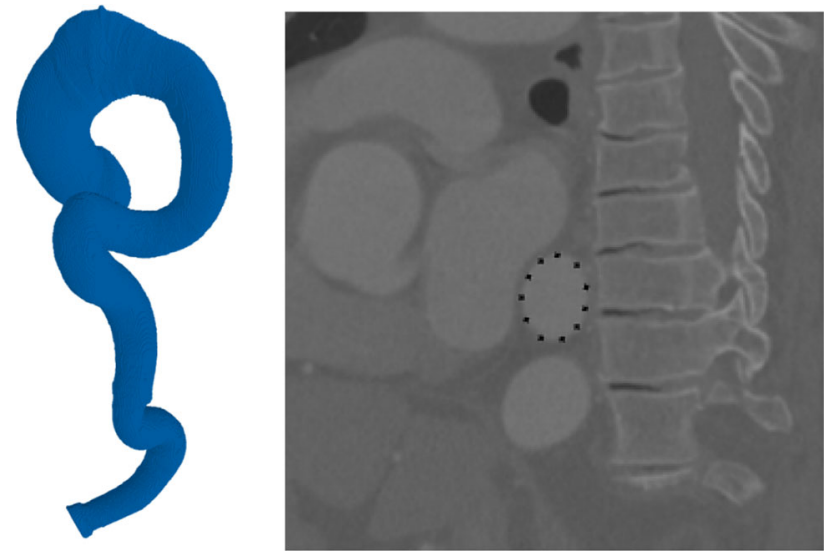

Fig. 4 Left: $3 D$ reconstruction of an aorta; right: one cross section of the aorta in the proximity of a severe elongation. The shape of this cross section approaches an ellipse

The output of the algorithm is the aorta centerline given by the points $\mathbf{c}_{\mathbf{k}}$, the orientation of each cross section, given by the angles $\left(\alpha_{k}, \beta_{k}\right)$ and the ellipses $C_{\mathbf{c}_{k}, \sigma}^{\alpha_{k}, \beta_{k}}$. From these data, it is possible to build a representation of the $3 D$ ellipses that model the cross sections calculated along the aorta (Fig. 6(left)). Therefore, what we obtain is not an aorta lumen segmentation in itself, i.e., we do not obtain a solid volume. However, for comparison purposes, we can easily obtain this solid volume by filling the inner volume between every consecutive pair of such ellipses (Fig. 6(right)). The intersection of such volume with axial planes enables the contour of the aorta in the original axial slices to be obtained.

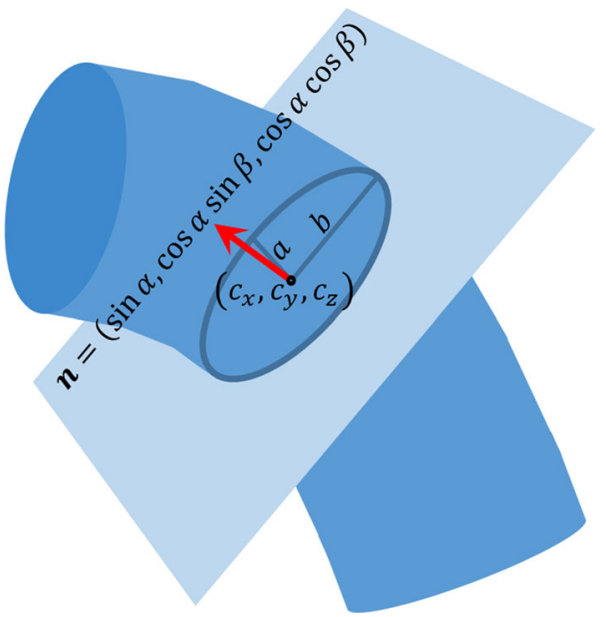

Fig. 5 Illustration of a cross-sectional plane including the centerline point $\mathbf{c}=\left(c_{x}, c_{y}, c_{z}\right)^{T}$, the plane normal orientation $\mathbf{n}$ given by the angles $\alpha, \beta$ and the ellipse corresponding to the intersection of the plane and the $3 D$ volume

\section{Experiments and dataset}

Images from two independent databases were employed to assess the robustness and the accuracy achieved by the algorithm. For robustness, a qualitative procedure based on visual inspection was designed. Thereby, the correct initialization of the algorithm and its capacity for tracking the whole aorta were analyzed. For accuracy, a quantitative evaluation procedure was performed. In this way, regions manually delineated by a human expert $\left(S_{\mathrm{m}}\right)$ were compared with regions extracted by the algorithm $\left(S_{\mathrm{a}}\right)$. 

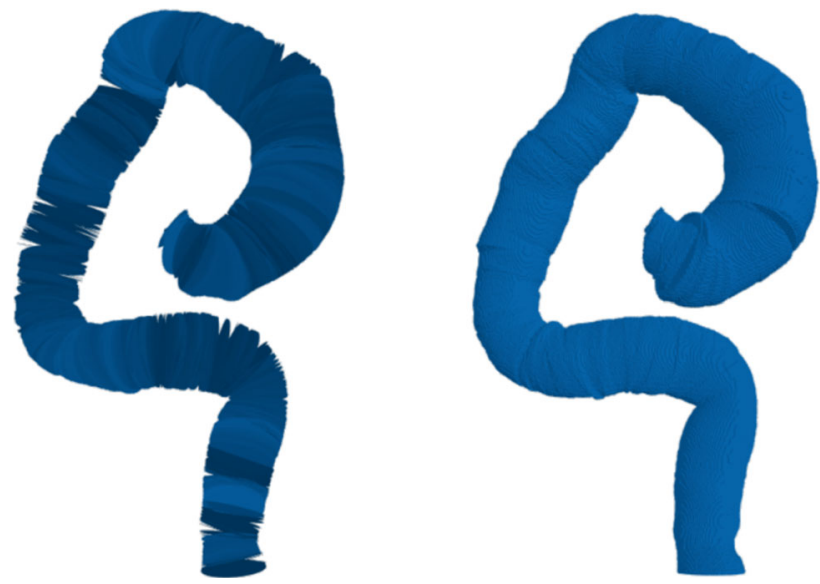

Fig. 6 Left: ellipses obtained for patient USC-119; right: automatic aorta volume segmentation computed from the ellipses

The first database, named USC, comprises 108 CT volumes of patients that were scanned in the Department of Radiology of the University Hospital of Santiago de Compostela, Spain. Normal and abnormal cases were included in this database. Most of the abnormal cases were related to the presence of aneurysms, but also different kinds of pathologies like dissections, mural thrombi, or severe elongations were included (Table 3). All the cases were obtained after intravenous contrast was injected, which is the standard protocol for this kind of patient. Thereby, the opacity of the lumen of the aorta is enhanced, which usually facilitates its detection. However, depending on the aortic illness that is suffered, the morphology of the lumen for these abnormal cases can be quite different to that expected, which makes it difficult to build a standard model. This database includes cases from two different scanners and the slice thickness ranges from 0.625 to $5.0 \mathrm{~mm}$. The dose is automatically adjusted depending on the physical characteristics of the patient.

The second database, named LIDC, comprises 277 CT volumes from the LIDC-IDRI public database (see $[18,19]$ ). Because of this database was built for lung cancer detection, most of the cases are normal as regards the aorta and were acquired as low-dose non-contrast CT images. This database includes cases from six different scanners and the slice thickness also ranges from 0.625 to $5.0 \mathrm{~mm}$.

Manual annotations were delineated using the paintbrush tool of the ITK-SNAP program (http://www.itksnap.org). A tablet with a digital stylus was employed for this task performed by a radiologist in collaboration with an experienced researcher (both are among the authors of this paper).

\section{Evaluation of the robustness}

Both the selection of the first contour and the final result achieved for the tracking procedure were analyzed. For the
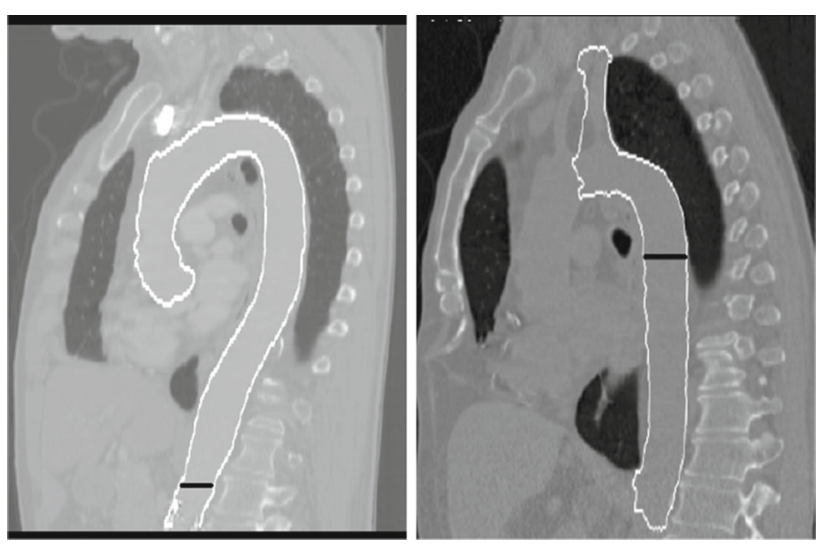

Fig. 7 Illustration (in white) of the intersection in the CT scan of the $3 \mathrm{D}$ ellipse collection with a plane approximating the centerline points. The dark segment inside the aorta represents the location of the contour used to initialize the tracking. Left: a CT scan where the method has successfully tracked the aortic lumen in all the aorta segments; Right: a CT scan where the method has failed to track the aortic lumen in the aortic arch

selection of the first contour, it was checked whether the candidate circles that the algorithm selected automatically were inside the lumen.

For the tracking procedure, we analyze, by visual inspection, whether the algorithm is able to properly track the aorta from the descending to the ascending aorta so that it does not get trapped in spurious locations. To do this, reformatted planes were obtained. In order to optimally visualize the shape of the aorta, those planes were calculated automatically using the centerline points (see Fig. 7).

\section{Evaluation of the accuracy}

Accuracy assessment was performed in two ways. (1) First, for each CT scan of the database, the algorithm provides a set of reformatted images where the cross sections of the aorta are placed (Fig. 10) in its center. For each case, we randomly selected eleven of those images (five located in the ascending aorta and the aortic arch, and six located in descending aorta) to manually trace the border of the aorta. (2) Second, to analyze the accuracy of the method in different contexts in more detail, 16 cases were specially selected to encompass the greatest possible variability. The aim was to analyze how accurate the algorithm with and without the presence of disease, artifacts and/or physiological contrast, etc. (see Table 3). For those cases, all the axial slices were delineated by hand. Thereby, the aorta volumes (manually extracted vs algorithm extracted) could be compared.

As comparative measures, we used the dice similarity coefficient (DSC) and the bias estimator $B_{p n}$ given by:

$\operatorname{DSC}=\frac{2\left|S_{\mathrm{m}} \cap S_{\mathrm{a}}\right|}{\left|S_{\mathrm{m}}\right|+\left|S_{\mathrm{a}}\right|}$, 


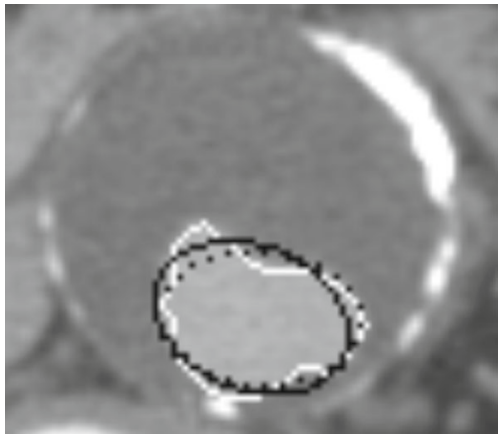

Fig. 8 Illustration of an aorta cross section with the lumen contour manually delineated (in white, $S_{\mathrm{m}}$ ), the ellipse estimated using the proposed method (black dotted line, $S_{\mathrm{a}}$ ) and the ellipse estimated from the manual contour (black continuous line, $S_{\mathrm{a}}^{1}$ )

$$
B_{p n}=\frac{\left|S_{\mathrm{a}} \backslash S_{\mathrm{m}}\right|-\left|S_{\mathrm{m}} \backslash S_{\mathrm{a}}\right|}{\left|S_{\mathrm{m}} \cap S_{\mathrm{a}}\right|} .
$$

We point out that when there is no overlap, the outcome of DSC is 0 , and for complete overlap the outcome is 1 . In the above expression for $B_{p n},\left|S_{\mathrm{a}} \backslash S_{\mathrm{m}}\right|$ and $\left|S_{\mathrm{m}} \backslash S_{\mathrm{a}}\right|$ compute the number of points that belong to $S_{\mathrm{a}}$ but are not within $S_{\mathrm{m}}$ and vice versa. Thus, $B_{p n}$ is positive (respectively, negative) in the case $S_{\text {a }}$ provides an over- (respectively, under- ) segmentation of $S_{\mathrm{m}}$

For each CT scan, we compare the region inside the manual segmentation $\left(S_{\mathrm{m}}\right)$, the region inside the estimated ellipse $\left(S_{\mathrm{a}}\right)$ and the region inside an ellipse estimated from the manual contour using a basic algebraic method to fit an ellipse to a collection of points (we name such region $S_{\mathrm{a}}^{1}$ ). In Fig. 8, we illustrate the shape of the contours of the sets $S_{\mathrm{m}}, S_{\mathrm{a}}$ and $S_{\mathrm{a}}^{1}$ for a particular cross section. We observe that this cross section is a challenging case because the aorta lumen does not have an elliptical shape due to a mural thrombus.

Furthermore, a $3 D$ segmentation of the aorta lumen is obtained for each selected CT by manual delineation of the lumen (in this case, this represents the set $S_{\mathrm{m}}$ ) and is compared with a 3D segmentation of the aortic lumen obtained by the proposed method (set $S_{\mathrm{a}}$ ). Figure 9 shows the sets $S_{\mathrm{a}}$ (blue) and $S_{\mathrm{m}}$ (red) for a particular CT. We also compute the Euclidean distance to the closest point in the boundary of the set $S_{\mathrm{m}}$ (Fig. 9 (right)) for every voxel in the boundary of the set $S_{\mathrm{a}}$.

\section{Results}

Figure 10 shows some of the ellipses computed by our method by minimizing the expression (8). The different structures of the CT volume appear represented in the projection and the calculated ellipses are overlaid to depict the behavior of the algorithm. We can observe that, even in the case of the presence of artifacts related to calcifications or metallic structures (stent), the robustness of the algorithm enables the aorta to be tracked, adapting the shape of the ellipse to the aortic wall up to the heart.

Figure 11 shows the 3D segmentations obtained for the sixteen selected cases. The colormap used is the same as in Fig. 9.

\section{Evaluation of the robustness}

After visual inspection, we conclude that all the circles selected for the sets of initial contours $\left\{C^{z_{i}}\right\}$ are inside the lumen for all the cases in the database. Moreover, in 380 out of 385 cases, the tracking procedure has provided centerline points passing through all aortic segments (the descending aorta, the aortic arch and the ascending aorta). In 351 out of these 380 cases where the tracking procedure does not get trapped in spurious locations, the method uses the first circle provided by the proposed method as initial contour to track the aorta. Thereby, in 29 cases the method has automatically detected that the tracking procedure has failed but has worked properly using another circle from the collection $\left\{C^{z_{i}}\right\}$ as initial contour.
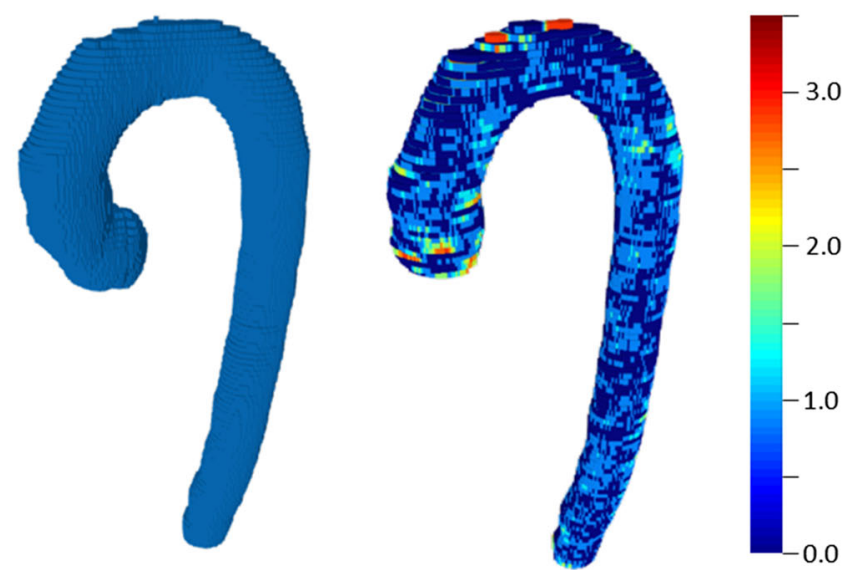

Fig. 9 Patient LIDC-0126. From left to right: manual segmentation (red), segmentation computed from the ellipses (blue), 3D distance map between both volumes. The colormap indicates the Euclidean distance to the closest point (in $\mathrm{mm}$ ) for every boundary voxel

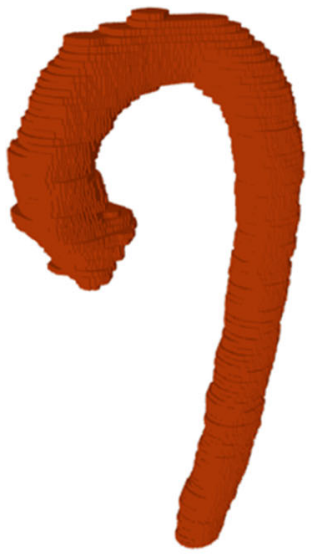



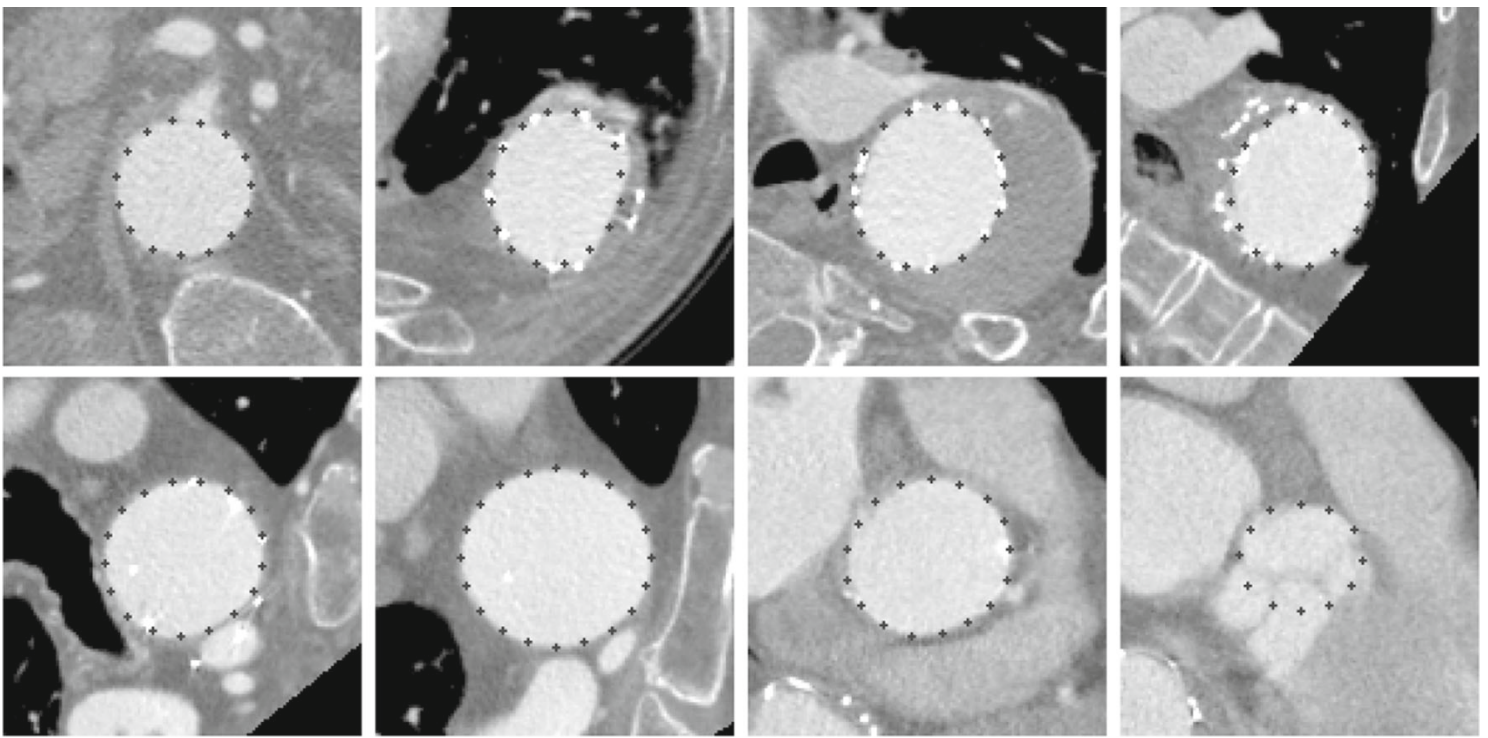

Fig. 10 Examples of aortic cross sections and the corresponding ellipses obtained for different segments of the aorta of patient USC-0119, using the proposed method. We observe that the patient has a stent surrounding part of the aortic lumen
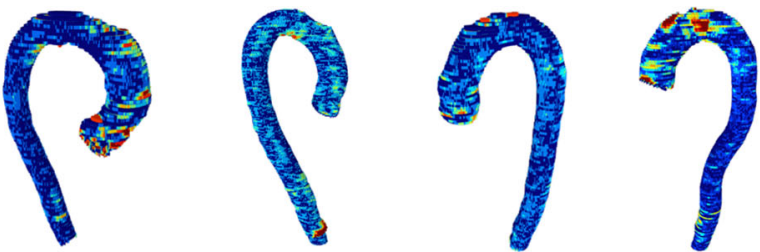

LIDC-0001

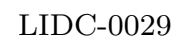

LIDC-0126
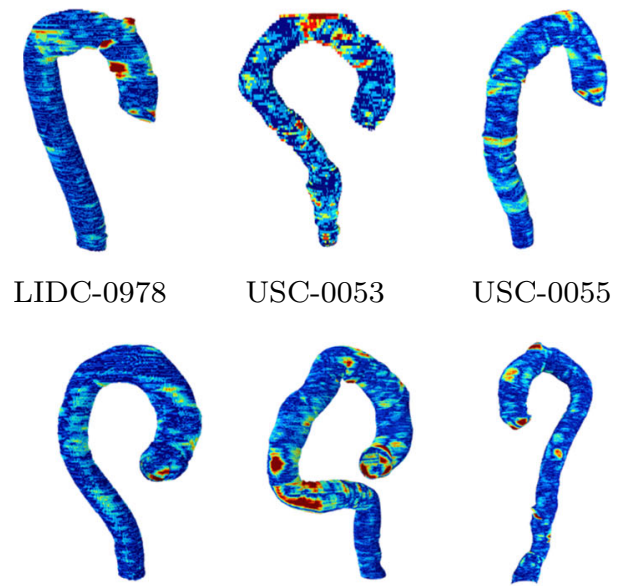

USC-0053

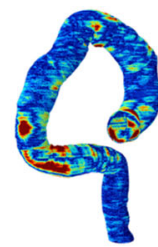

USC-0104
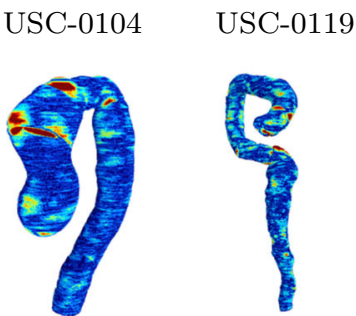

USC-0149

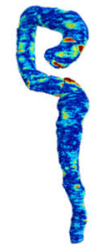

USC-0164
USC-0055

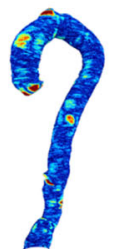

USC-0139

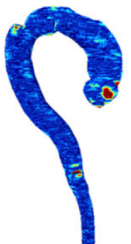

USC-0173
LIDC-0836

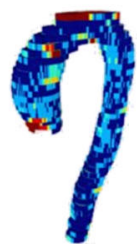

USC-0100

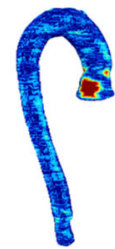

USC-0141

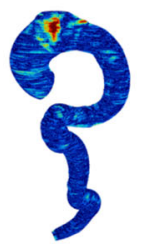

USC-EL01

Fig. 11 3D segmentations for the sixteen selected cases. The colormap used is the same as in Fig. 9

\section{Evaluation of the accuracy}

Tables 1 and 2 depict a summary of the results achieved for quantitative evaluation for the entire database. The mean, and some percentiles of the DSC and $B_{p n}$ estimators were computed. DSC ${ }^{1}$ and $B_{p n}^{1}$ correspond to the comparison between $S_{\mathrm{m}}$ and $S_{\mathrm{a}}^{1}$. We separate the study according to the location of the cross sections in the different segments of the aorta. We also show the eccentricities of the ellipses given by the boundary of the set $S_{\mathrm{a}}$ (named ECC) and the boundary of the set $S_{\mathrm{a}}^{1}\left(\right.$ named ECC $\left.{ }^{1}\right)$.

Table 3 shows the DSC and $B_{p n}$ estimators for the sixteen selected cases. It also includes the mean and several percentiles of the distribution of the distances between boundary voxels, as described above.

\section{Discussion}

The results obtained demonstrate that the developed procedure is able to extract the aortic geometry from normal

Table 1 Results of the quantitative evaluation for ascending aorta and aortic arch segments

\begin{tabular}{lccccc}
\hline & Mean & $P_{0.01}$ & $P_{0.05}$ & $P_{0.25}$ & $P_{0.5}$ \\
\hline DSC & 0.953 & 0.829 & 0.909 & 0.945 & 0.959 \\
DSC $^{1}$ & 0.972 & 0.941 & 0.958 & 0.969 & 0.973 \\
$B_{p n}$ & -0.04 & -0.30 & -0.15 & -0.07 & -0.03 \\
$B_{p n}^{1}$ & -0.05 & -0.08 & -0.07 & -0.05 & -0.05 \\
ECC $^{1}$ & 0.238 & 0.000 & 0.000 & 0.068 & 0.247 \\
ECC $^{1}$ & 0.265 & 0.000 & 0.000 & 0.136 & 0.279 \\
\hline
\end{tabular}


Table 2 Results of the quantitative evaluation for descending aorta

\begin{tabular}{lccccc}
\hline & Mean & $P_{0.01}$ & $P_{0.05}$ & $P_{0.25}$ & $P_{0.5}$ \\
\hline DSC & 0.950 & 0.840 & 0.911 & 0.942 & 0.956 \\
DSC $^{1}$ & 0.967 & 0.938 & 0.953 & 0.964 & 0.969 \\
$B_{p n}$ & -0.05 & -0.29 & -0.15 & -0.08 & -0.04 \\
$B_{p n}^{1}$ & -0.06 & -0.10 & -0.09 & -0.07 & -0.06 \\
ECC $^{1}$ & 0.181 & 0.000 & 0.000 & 0.012 & 0.151 \\
ECC $^{1}$ & 0.222 & 0.000 & 0.000 & 0.072 & 0.218 \\
\hline
\end{tabular}

and abnormal CT volumes acquired at different conditions (including low-dose and non-contrast) in more than $98 \%$ of the cases, and even in situations where the presence of artifacts as part of the image is relevant.

Inspection of Tables 1 and 2 shows that the DSC has mean values above 0.95 for all the aorta sections. Even at relatively low percentiles $P_{0.05}$, those values are still higher than 0.9 , which means that there is a high degree of similarity between manual and automatic aortic lumen extractions. Moreover, those values are even higher for $\mathrm{DSC}^{1}$, which demonstrates that modeling the aortic lumen as an ellipse seems to be a valid assumption. This is confirmed by analyzing the values achieved by $E C C$, where a certain degree of eccentricity is demonstrated, which is more relevant for the ascending aorta and the aortic arch.

Table 3 Results of the quantitative evaluation for the sixteen selected cases. For pathology, A refers to the presence of aneurysms, $\mathrm{C}$ refers to the presence of calcifications, D refers to the presence of dissections, MT refers to the presence of mural thrombi, E refers to the presence of elongations, $\mathrm{N}$ refers to normal case and $\mathrm{S}$ refers to the presence
On the other hand, $B_{p n}$ is slightly biased to negative values, but close to zero (see Tables 1 and 2). Hence, no significant differences between the regions are selected by both methods, except for a few cases (less than $1 \%$ ), where the region selected by the automatic method tends to be smaller than the manually selected one (see $P_{0.01}$ ).

From the analysis of the results shown in Tables 1 and 2, we can also conclude that there is no significant differences for DSC and $B_{p n}$ as regards the position where the algorithm was applied (descending aorta vs ascending aorta and aortic arch). No leakages were observed, and the algorithm was able to correct the ellipse orientation and continue tracking the aorta properly, in most of the cases. We have observed that the presence of lateral vessels like the carotid arteries (aortic arch) or the renal artery (descending aorta) can reduce, in some cases, the accuracy of the algorithm generating, locally, high variation in the aorta cross-sectional plane orientation. This may introduce some small indentations when the solid volume is generated. However, this only happens for a small number of slices, which is not significant in terms of the computation of the whole CT volume. In any case, as a postprocessing step, active contour models could be used to address this issue.

The analysis of Table 3 shows that, for the sixteen selected cases, the mean distance is lower than $0.9 \mathrm{~mm}$. Considering that the normal diameter of the aorta typically ranges from 30 to $40 \mathrm{~mm}$, we can conclude that the algorithm has performed of stents. DSC and $B_{p n}$ are computed between the estimated and the manually delineated aorta segmentations. The mean and the percentiles $P_{0.5}, P_{0.75}, P_{0.95}, P_{0.99}$ are computed for the distribution of distances (in $\mathrm{mm}$ ) between the voxels of the contours of both segmentations

\begin{tabular}{|c|c|c|c|c|c|c|c|c|c|}
\hline Case & Pathology & Voxel size $(\Delta x, \Delta y, \Delta z)$ & DSC & $B_{p n}$ & Mean distance (mm) & $P_{0.5}$ & $P_{0.75}$ & $P_{0.95}$ & $P_{0.99}$ \\
\hline LIDC-0001 & $\mathrm{N}, \mathrm{C}$ & $(0.703,0.703,2.500)$ & 0.928 & -0.073 & 0.546 & 0.000 & 0.703 & 2.223 & 2.899 \\
\hline LIDC-0029 & $\mathrm{N}, \mathrm{C}$ & $(0.879,0.879,1.250)$ & 0.954 & -0.033 & 0.581 & 0.879 & 0.879 & 1.250 & 2.157 \\
\hline LIDC-0126 & $\mathrm{N}$ & $(0.781,0.781,2.500)$ & 0.959 & -0.027 & 0.387 & 0.000 & 0.781 & 1.105 & 2.471 \\
\hline LIDC-0836 & $\mathrm{N}$ & $(0.547,0.547,1.800)$ & 0.928 & -0.023 & 0.661 & 0.547 & 0.773 & 2.373 & 3.981 \\
\hline LIDC-0978 & $\mathrm{N}, \mathrm{C}$ & $(0.725,0.725,0.750)$ & 0.947 & -0.001 & 0.590 & 0.725 & 0.725 & 1.632 & 3.129 \\
\hline USC-0053 & $\mathrm{A}, \mathrm{MT}$ & $(0.873,0.873,2.500)$ & 0.932 & -0.037 & 0.672 & 0.873 & 0.873 & 2.469 & 3.049 \\
\hline USC-0055 & S & $(0.730,0.730,0.620)$ & 0.942 & -0.012 & 0.691 & 0.620 & 0.958 & 1.633 & 2.585 \\
\hline USC-0100 & $\mathrm{C}$ & $(0.910,0.910,5.000)$ & 0.936 & 0.022 & 0.625 & 0.000 & 0.910 & 3.641 & 5.000 \\
\hline USC-0104 & $\mathrm{N}$ & $(0.865,0.865,0.625)$ & 0.934 & -0.100 & 0.569 & 0.625 & 0.865 & 1.520 & 2.065 \\
\hline USC-0119 & $\mathrm{E}, \mathrm{S}$ & $(0.758,0.758,0.620)$ & 0.949 & -0.16 & 0.810 & 0.620 & 0.979 & 2.399 & 3.871 \\
\hline USC-0139 & $\mathrm{C}, \mathrm{MT}$ & $(0.775,0.775,0.620)$ & 0.947 & -0.043 & 0.608 & 0.620 & 0.775 & 1.655 & 3.877 \\
\hline USC-0141 & $\mathrm{N}$ & $(0.854,0.854,0.620)$ & 0.941 & -0.103 & 0.550 & 0.620 & 0.854 & 1.207 & 2.758 \\
\hline USC-0149 & $\mathrm{A}, \mathrm{C}$ & $(0.703,0.703,0.620)$ & 0.961 & -0.034 & 0.624 & 0.620 & 0.703 & 1.589 & 4.219 \\
\hline USC-0164 & MT & $(0.912,0.912,0.625)$ & 0.935 & -0.050 & 0.756 & 0.625 & 1.106 & 1.928 & 3.966 \\
\hline USC-0173 & $\mathrm{D}$ & $(0.703,0.703,0.620)$ & 0.950 & -0.049 & 0.509 & 0.620 & 0.703 & 1.240 & 3.372 \\
\hline USC-EL01 & $\mathrm{E}$ & $(0.703,0.703,0.620)$ & 0.966 & -0.011 & 0.442 & 0.620 & 0.620 & 0.994 & 1.690 \\
\hline
\end{tabular}


an accurate segmentation in both databases (USC and LIDC) regardless of the presence of different kinds of abnormalities.

From the analysis of the percentiles in Table 3, we can state that such values are very low in general. For three cases, $P_{0.5}$ is exactly 0.0 . This means that most of the boundary voxels obtained by the automatic procedure match the manually marked boundary. Furthermore, for all the cases, $P_{0.75}$ is lower than $1.2 \mathrm{~mm}$. Regarding the values of $P_{0.95}$ and $P_{0.99}$, those relatively high numbers $(>3 \mathrm{~mm})$ are related either with the presence of artifacts or with the presence of small indentations in the solid volume, generated as described above. In this sense, the role of the voxel size should be highlighted. The larger the voxel, the greater the indentation. Therefore, low-resolution CT cases may be more affected by this drawback.

However, we have to point out that a fair quantitative comparison with other published studies is not possible because the image databases used are not available in these studies. In fact, as a contribution of our paper we provide the database we have used, including annotated aorta segmentations as well as an implementation of our algorithm so that anyone can reproduce our experiments or test their own algorithms in the same image database. We also point out that, among the images we selected for our study, there are a number of challenging ones including patients suffering severe elongations, aneurysms, calcifications, stent implantations or low contrast media administration. From a quantitative point of view, in our database we obtain a mean DSC of 0.951 for the 380 cases we tested ( 0.928 for the 16 selected cases). Using different databases, Kurugol et al. [6] reported a mean DSC of 0.92 (using 45 CT cases). Xie et al. [8] reported a mean DSC of 0.933 on a dataset of $60 \mathrm{CT}$ cases. On the other hand, the method proposed in this paper does not require the development of any complex model for the aorta, as is the case for Isgun et al. [20]. This avoids the need to perform time-consuming multiple registrations (around $15 \mathrm{~min}$ for a single registration in that case). In this sense, it is worth mentioning that the dynamic programming method proposed by Avila-Montes et al. [21] takes around $9 \mathrm{~min}$. In our case, all calculations take less than $1 \mathrm{~min}$, on a standard $\mathrm{PC}$, for a typical CT case with 500 slices. Furthermore, our method does not need the prior detection of any anatomic landmark to start the process, unlike in Xie et al. [8] and Kurugol et al. [6], which require detecting the carina.

\section{Conclusions}

A fully automated algorithm for the extraction of the aorta centerline, cross sections and their corresponding ellipses fitting the aorta lumen contours has been developed for chest CT volumes, and evaluated using $385 \mathrm{CT}$ cases from two different databases. The algorithm that models the cross section of the aorta as an ellipse is able to track the whole structure of the aorta by adapting the ellipse to the best size, eccentricity, and $3 \mathrm{D}$ orientation.

The algorithm successfully segmented the aorta in most of the cases. By visual inspection, about $98 \%$ of the cases were successfully segmented. Only in 5 out of 385 cases was the algorithm unable to fully track the aorta, because the algorithm gets trapped in the aortic arch. The results achieved by the algorithm for the entire databases in terms of mean DSC were 0.951 (0.953 for descending aorta, and 0.950 for ascending aorta and aortic arch). The DSC computed for the 3D segmentations of the selected $16 \mathrm{CT}$ cases is always higher than 0.928 . The mean distance was less than $0.9 \mathrm{~mm}$ for the sixteen selected cases. We have observed that the method is robust and accurate and can provide good results for normal and abnormal cases, even in challenging situations or in regions close to the heart, where the presence of different kinds of artifacts may affect the performance of the algorithm.

Finally, as part of our contribution, we would like to mention that we have included the software and the dataset that was used as experimental setup on the website http://www. ctim.es/demo110/, for reproducibility purposes.

Acknowledgements This research has partially been supported by the MINECO projects references TIN2016-76373-P (AEI/FEDER, UE) and MTM2016-75339-P (AEI/FEDER, UE) (Ministerio de Economía y Competitividad, Spain).

\section{Compliance with ethical standards}

Conflict of interest The authors declare that they have no conflict of interest.

Ethical approval All procedures performed in studies involving human participants were in accordance with the ethical standards of the institutional and/or national research committee and with the 1964 Helsinki Declaration and its later amendments or comparable ethical standards. For this type of study, formal consent is not required.

\section{References}

1. Sackett DL, Rosenberg W, Mc Gray JA, Haynes RB, Richardson WS (1996) Evidence-based medicine: what it is and what it isn't. BMJ 312:71-2

2. Hiratzka LF, Bakris GL, Beckman JA, Bersin RM, Carr VF, Casey DE, Eagle KA, Hermann LK, Isselbacher EM, Kazerooni EA, Kouchoukos NT, Lytle BW, Milewicz DM, Reich DL, Sen S, Shinn JA, Svensson LG, Williams DM (2010) 2010 ACCF/AHA/AATS/ACR/ASA/SCA/SCAI/SIR/ STS/SVM Guidelines for the diagnosis and management of patients with thoracic aortic disease. J Am Coll Cardiol 55(14):1509-1544

3. Lesage D, Angelini E, Bloch I, Funka-Lea G (2009) A review of $3 \mathrm{D}$ vessel lumen segmentation techniques: models, features and extraction schemes. Med Image Anal 13(6):819-845

4. Wang S, Fu L, Yue Y, Kang Y, Liu J (2009) Fast and automatic segmentation of ascending aorta in MSCT volume data. In: 2nd 
International congress on image and signal processing (CISP), pp $1-5$

5. Martinez-Mera JA, Tahoces PG, Carreira JM, Suárez-Cuenca JJ, Souto M (2013) A hybrid method based on level set and 3D region growing for segmentation of the thoracic aorta. Comput Aided Surg 18(5-6):109-117

6. Kurugol S, Come CE, Diaz AA, Ross JC (2015) Automated quantitative $3 \mathrm{D}$ analysis of aorta size, morphology, and mural calcification distributions. Med Phys 42(9):5467-5478

7. Dasgupta A, Mukhopadhyay S, Mehre SA, Bhattacharyya P (2016) Morphological geodesic active contour based automatic aorta segmentation in thoracic CT images. In: International conference on computer vision and image processing (CVIP), pp 187-196

8. Xie Y, Padgett J, Biancardi AM, Reeves AP (2014) Automated aorta segmentation in low-dose chest CT images. Int J Comput Assoc Radiol 9:211-219

9. Krissian K, Malandain G, Ayache N, Vaillant R, Trousset Y (2000) Model-based detection of tubular structures in 3D images. Comput Vis Image Underst 80(2):130-171

10. Frangi AF, Niessen WJ, Vincken KL, Viergever MA (1998) Multiscale vessel enhancement filtering. In: International conference on medical image computing and computer-assisted intervention, pp 130-137

11. Alvarez L, Trujillo A, Cuenca C, González E, Esclarín J, Gomez L, Mazorra L, Alemán-Flores M, Tahoces PG, Carreira JM (2017) Tracking the aortic lumen geometry by optimizing the 3D orientation of its cross-sections. In: International conference on medical image computing and computer-assisted intervention (MICCAI 2017) LNCS, vol 10434, pp 174-181

12. Alvarez L, González E, Cuenca C, Trujillo A, Tahoces PG, Carreira JM (2018) Ellipse motion estimation using parametric snakes. J Math Imaging Vis 60(7):1095-1110

13. Elefteriades JA, Farkas EA (2010) Thoracic aortic aneurysm. Clinical pertinent controversies and uncertainties. J Am Coll Cardiol 55(9):841-857

14. Rajiah P, Shchoenhagen P (2013) The role of computed tomography in pre-procedural planning of cardiovascular surgery and intervention. Insights Imaging 4:671-689
15. Redheuil A, Yu W-C, Mousseaux E, Harouni AA, Kachenoura N, Wu CO, Bluemke D, Lima JAC (2011) Age-related changes in aortic arch geometry relationship with proximal aortic function and left ventricular mass and remodeling. J Am Coll Cardiol 58(12):12621270

16. Alvarez L, González E, Esclarín J, Gomez L, Alemán-Flores M, Trujillo A, Cuenca C, Mazorra L, Tahoces PG, Carreira JM (2017) Robust detection of circles in the vessel contours and application to local probability density estimation. In: CVII-STENT workshop proceedings, held in conjunction with MICCAI 2017, LNCS, vol 10552, pp 3-11

17. Cuenca C, González E, Trujillo A, Esclarín J, Mazorra L, Alvarez L, Martínez-Mera JA, Tahoces PG, Carreira JM (2018) Fast and accurate circle tracking using active contour models. J Real-Time Image Process 14:793-802

18. Armato SG, McLennan G, Bidaut L, McNitt-Gray MF, Meyer CR, Reeves AP, Zhao B, Aberle DR, Henschke CI, Hoffman EA, Kazerooni EA, MacMahon H, Van Beeke EJ, Yankelevitz D, Biancardi AM, Bland PH, Brown MS, Engelmann RM, Laderach GE, Max D, Pais RC, Qing DP, Roberts RY, Smith AR, Starkey A, Batrah P, Caligiuri P, Farooqi A, Gladish GW, Jude CM, Munden RF, Petkovska I, Quint LE, Schwartz LH, Sundaram B, Dodd LE, Fenimore C, Gur D, Petrick N, Freymann J, Kirby J, Hughes B, Casteele AV, Gupte S, Sallamm M, Heath MD, Kuhn MH, Dharaiya E, Burns R, Fryd DS, Salganicoff M, Anand V, Shreter U, Vastagh S, Croft BY (2011) The Lung Image Database Consortium (LIDC) and Image Database Resource Initiative (IDRI): a completed reference database of lung nodules on CT scans. Med Phys 38(2):915-931

19. Clark K, Vendt B, Smith K, Freymann J, Kirby J, Koppel P, Moore S, Phillips S, Maffitt D, Pringle M, Tarbox L, Prior F (2013) The cancer imaging archive (TCIA): maintaining and operating a public information repository. J Digit Imaging 26(6):1045-1057

20. Isgum I, Staring M, Rutten A, Prokop M, Viergever M, Van Ginneken B (2009) Multi-atlas-based segmentation with local decision fusion application to cardiac and aortic segmentation in CT scans. IEEE Trans Med Imag 28(7):1000-1010

21. Avila-Montes OC, Kurkure U, Nakazato R, Berman DS, Dey D, Kakadiaris IA (2013) Segmentation of the thoracic aorta in noncontrast cardiac CT images. IEEE J Biomed Health Inform 17(5):936-49 